ARTICLE

Received 7 Jun 2012 | Accepted 2 Oct 2012 | Published 13 Nov $2012 \quad$ DOl: 10.1038/ncomms2184

\title{
Structural modelling and mutant cycle analysis predict pharmacoresponsiveness of a $\mathrm{Na}_{\mathrm{v}} 1.7$ mutant channel
}

Yang Yang 1,2,3, Sulayman D. Dib-Hajj ${ }^{1,2,3}$, Jian Zhang ${ }^{4}$, Yang Zhang ${ }^{4}$, Lynda Tyrrell 1,2,3, Mark Estacion ${ }^{1,2,3}$ \& Stephen G. Waxman 1,2,3

Sodium channel Nav1.7 is critical for human pain signalling. Gain-of-function mutations produce pain syndromes including inherited erythromelalgia, which is usually resistant to pharmacotherapy, but carbamazepine normalizes activation of Nav1.7-V400M mutant channels from a family with carbamazepine-responsive inherited erythromelalgia. Here we show that structural modelling and thermodynamic analysis predict pharmacoresponsiveness of another mutant channel (S241T) that is located 159 amino acids distant from V400M. Structural modelling reveals that $\mathrm{Na}_{\mathrm{v}} 1.7-\mathrm{S} 241 \mathrm{~T}$ is $\sim 2.4 \AA$ apart from V400M in the folded channel, and thermodynamic analysis demonstrates energetic coupling of V400M and S241T during activation. Atomic proximity and energetic coupling are paralleled by pharmacological coupling, as carbamazepine ( $30 \mu \mathrm{M})$ depolarizes S214T activation, as previously reported for V400M. Pharmacoresponsiveness of S241T to carbamazepine was further evident at a cellular level, where carbamazepine normalized the hyperexcitability of dorsal root ganglion neurons expressing S241T. We suggest that this approach might identify variants that confer enhanced pharmacoresponsiveness on a variety of channels.

\footnotetext{
${ }^{1}$ Department of Neurology, Yale University School of Medicine, New Haven, Connecticut 06510, USA. ${ }^{2}$ Center for Neuroscience \& Regeneration Research, Yale University School of Medicine, New Haven, Connecticut 06510, USA. ${ }^{3}$ Rehabilitation Research Center, VA Connecticut Healthcare System, West Haven, Connecticut 06516, USA. ${ }^{4}$ Center for Computational Medicine and Bioinformatics, University of Michigan, 100 Washtenaw Avenue, Ann Arbor, Michigan 48109, USA. Correspondence and requests for materials should be addressed to S.G.W. (email: stephen.waxman@yale.edu).
} 
C hronic pain affects more than one-quarter of Americans and pain in nearly $40 \%$ of these patients is not relieved by currently available drugs ${ }^{1}$. Recent studies have demonstrated that $\mathrm{Na}_{\mathrm{V}} 1.7$, a voltage-gated sodium channel preferentially expressed in dorsal root ganglia (DRG) and sympathetic ganglia neurons, is essential for pain transduction ${ }^{2-5}$. Loss-of-function $\mathrm{Na}_{\mathrm{V}} 1.7$ mutations cause congenital indifference to pain $^{6}$, whereas gain-of-function mutations produce several painful syndromes including inherited erythromelalgia (IEM), a disorder in which, as a result of hyperpolarized activation of $\mathrm{Na}_{V}$ 1.7, threshold is reduced and evoked firing frequency increased in pain-signalling DRG neurons, leading to intense burning pain ${ }^{4,7,8}$. These studies have validated $\mathrm{Na}_{\mathrm{V}} 1.7$ as a molecular target for development of new pain therapeutics.

IEM is characteristically unresponsive to existing pharmacotherapies $^{7}$. Against this background of pharmaco-unresponsiveness, multiple-affected members of one family, carrying the $\mathrm{Na}_{V} 1.7$ V400M mutation, have responded favourably to carbamazepine $(\mathrm{CBZ})^{9}$, a state-dependent sodium channel blocker used mainly for treatment of epilepsy and trigeminal neuralgia ${ }^{10}$. Our previous study demonstrated that therapeutic concentrations of CBZ normalize the hyperpolarizing shift of $\mathrm{Na}_{\mathrm{V}} 1.7-\mathrm{V} 400 \mathrm{M}$ mutant channel activation', while not affecting activation of wild-type channels. As the shift of activation voltage dependence is a strong contributor to hyperexcitability of DRG neurons expressing IEM mutations $^{11}$, normalization of activation in V400M by CBZ seems to underlie the beneficial effect of CBZ in this family? 9 . These findings suggest the feasibility of personalized pharmacotherapybased upon a pharmacogenomic approach. To achieve this goal, better understanding of structure-function relationships of human $\mathrm{Na}_{V} 1.7$ channel mutations that underlie drug responsiveness and development of pre-clinical assays of pharmacoresponsiveness are needed.

The first demonstration of the crystal structure of an ion channel, KcsA ${ }^{12}$, provided a basis for structural modelling of the ion channel superfamily. Recently, the crystal structure of the bacterial voltage-gated sodium channel $\mathrm{NavAb}$ has been solved, shedding new light on structural substrates for channel gating and drug accessibility ${ }^{13}$. Starting from modelling of the human $\mathrm{Na}_{\mathrm{V}} 1.7$ channel, we demonstrate in this study that an approach combining structural modelling, thermodynamic mutant cycle analysis and voltage- and currentclamp recording of $\mathrm{Na}_{\mathrm{V}} 1.7$ mutant channels can predict pharmacoresponsiveness of a $\mathrm{Na}_{\mathrm{V}} 1.7$ mutant channel (S241T) to CBZ, suggesting a novel approach to screening for amino-acid variants that enhance pharmacoresponsiveness of a variety of channels.

\section{Results}

V400 and S241 show atomic proximity in human $\mathrm{Na}_{\mathrm{V}} 1.7$ channel. Previous observations from the primary protein sequence suggest that IEM mutations of $\mathrm{Na}_{\mathrm{V}} 1.7$ tend to cluster in transmembrane segments and S4-S5 linkers of domains I-III (ref. 4) but their proximity to each other within the folded structure is not clear. To better understand structure-function relationships among IEM mutations, we constructed a structural model of transmembrane helices of human $\mathrm{Na}_{\mathrm{V}} 1.7$ channel based on the recently solved crystal structure of the bacterial voltagegated sodium channel $\mathrm{NavAb}^{13}$.

The human $\mathrm{Na}_{\mathrm{V}} 1.7$ structural model was constructed using the GPCR-ITASSER program, an extension of the well-established I-TASSER algorithm ${ }^{14,15}$, incorporating a composite set of transmembrane-specific force fields and constraints ${ }^{16}$. Accuracy of individual domain models can be reliably estimated by the confidence score (C-score), which has a high correlation to the actual TM-score ${ }^{17}$, a widely used measure of similarity between two protein structures with values in the $[0,1]$ range. Unlike RMSD (root-mean-square-deviation) score, TM-score is insensitive to local structure variation and protein size. In general, two structures with TM-scores $<0.17$ correspond to random similarity and those with TM-scores $>0.5$ indicate high similarity between the predicted model and native structure ${ }^{18,19}$. Estimated TM-scores based on C-scores for our models of domains I-IV were $0.575,0.805,0.681$ and 0.722 , respectively, indicating similar folding structures to those of the bacterial sodium channel.

The linear schematic of $\mathrm{Na}_{\mathrm{V}} 1.7$ channel is shown in Fig. 1a. Assembled folded structural models are shown in Fig. 1b (intramembrane side view) and Fig. 1c (cytosolic view). We are interested in the V400M mutation of DI/S6 helix because it is known to be pharmacoresponsive to $\mathrm{CBZ}^{9}$. Reasoning that location of $\mathrm{V} 400 \mathrm{M}$ in the folded channel might be critical for its pharmacoresponsiveness, we looked for IEM mutations that are structurally close to V400M. Interestingly, our structural model revealed that the location of another IEM mutation (S241T) of $\mathrm{DI} / \mathrm{S} 4-\mathrm{S} 5$ linker is close to that of V400M. The distance between S241 and V400 is only $2.4 \AA$ measured from the closest hydrogen in the folded channel. This proximity, however, does not necessarily mean that the two residues engage in a direct interaction. Zoom-in views of DI/S6 show locations of V400 and S241 (Fig. 1d,e). The V400 side chain points toward the S4-S5 linker, where S241 is located (Fig. 1d,e). Although the effect of S241T on channel activation has been linked to increased size of the side chain at position 241 (probably through a steric hindrance effect) ${ }^{20}$, pharmacoresponsiveness of S241T mutant channel has not been previously assessed.

Another IEM mutation $(\mathrm{F} 1449 \mathrm{~V})^{21}$ was selected for analysis because it is located at the cytoplasmic end of S6 helix, which brings it into proximity with the V400M mutation in DI/S6 (Fig. 1d,e). Based on homology modelling to potassium channel structure, F1449 has been suggested to contribute to a hydrophobic ring at the cytoplasmic pore opening, which acts as an activation gate ${ }^{22}$. Modelling of F1449 using the bacterial sodium channel structure recapitulated the hydrophobic ring. However, like the S241T mutant, the pharmacoresponsiveness of F1449V mutant channel is unknown.

V400M and S241T are energetically coupled during activation.. Previous studies show that all of these IEM mutations hyperpolarize channel activation ${ }^{4,9,20-22}$. However, it is not clear whether these mutations work independently or in concert to affect the same or different aspect of activation. We therefore asked whether the location of $\mathrm{Na}_{\mathrm{V}} 1.7$ channel mutations demonstrated by our structural modelling might indicate mechanistic coupling during channel activation.

Thermodynamic mutant cycle analysis is a well-established approach for measuring independence or coupling of two mutations of a given protein ${ }^{23,24}$. We studied the coupling of V400M, a mutation located in the S6 helix, with either a structurally close mutation in the S4-S5 linker (S241T) or with a mutation in a different S6 helix (F1449V). Constructs carrying double mutations, for example, V400M and S241T or V400M and F1449V (named VM/ST and VM/FV, respectively) were created for mutant cycle analysis. Voltage dependence of activation was determined for $\mathrm{Na}_{\mathrm{V}} 1.7$ WT channel, single mutations (V400M, F1449V and S241T) and double mutations (VM/ST and VM/FV) in transiently transfected HEK293 cells. If two mutations are energetically coupled (or affect channel activation through the same or shared mechanism), then the double mutation would not have an additive effect on channel activation compared with the 
a
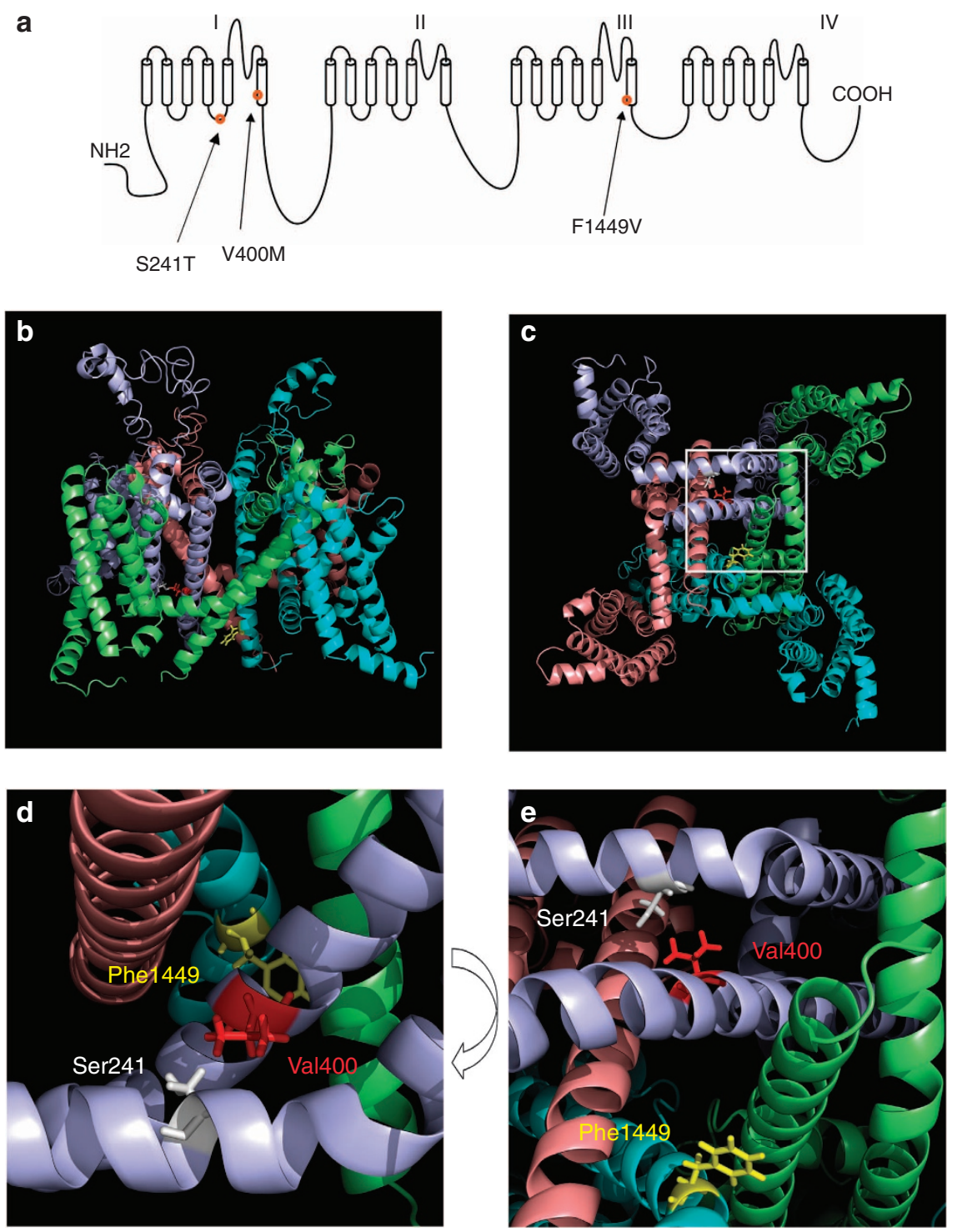

Figure 1 | Structural modelling of transmembrane domains of human Nav1.7 channel. (a) Schematic of the human Nav1.7 channel topology showing the mutations S241T, V400M and F1449V. (b) Intra-membrane view of structural model of Nav1.7 channel transmembrane domains. Domain I, light blue; Domain II, salmon; Domain III, cyan; Domain IV, lime. (c) Cyotsolic view of the structural model of Nav1.7 channel transmembrane domains. Boxed area containing S241, V400 and F1449 residues is enlarged in panel e. (d) Close-up intra-membrane view of the area containing S241, V400 and F1449 residues. (e) Close-up cytosolic view of the boxed area of panel c. S241, V400 and F1449 are shown as stick and coloured grey, red and yellow, respectively.

single mutations. However, if two mutations are not energetically coupled (or affect the channel independently through distinct mechanisms), then the double mutation would have an additive effect on channel activation compared with the two single mutations.

The voltage dependence of activation for WT, the mutant pairs to be evaluated and the corresponding double mutant were fitted with the Boltzmann equation to determine the $V_{1 / 2}$ (halfactivation voltage) and $Z$ (proportional to the slope at half activation)(Fig. 2a,b). Representative traces are shown in Fig. 2c-h. Activation of double mutation VM/ST was rather close to that of the single mutations, indicating a non-additive effect and suggesting that $\mathrm{V} 400 \mathrm{M}$ and S241T mutations alter activation via a common, shared mechanism (Fig. 2a). In contrast, activation of double mutation $\mathrm{VM} / \mathrm{FV}$ was more hyperpolarized relative to the single mutations (Fig. 2b), indicating an additive effect and suggesting that the V400M and F1449V mutations alter activation via different, energetically independent mechanisms.

To quantitatively assess these effects, the energy change on channel activation caused by a single or double mutation was calculated. For each single or double mutation, $V_{1 / 2}$ and $Z$ were used to determine free energy of activation $\left(\Delta G^{\circ}\right)$. The change in free energy of mutants relative to the WT channel was designated as $\left(\Delta \Delta G^{\circ}\right)$ and coupling free energy (magnitude of non-additivity) was $\left(\sum \Delta G^{\circ}\right)$. Designation and calculation of $\left(\Delta \Delta G^{\circ}\right)$ and $\left(\sum \Delta G^{\circ}\right)$ are described in Methods. Based on previous studies ${ }^{23-28}$, we set the following standard: if $\left|\sum \Delta G^{\circ}\right|>1 \mathrm{kcal} \mathrm{mol}^{-1}$, we accepted the two mutations as coupled (non-additive); whereas if $\left|\sum \Delta G^{\circ}\right|<1 \mathrm{kcal} \mathrm{mol}^{-1}$, we accepted the two mutations as independent (additive). We calculated $\sum \Delta G^{\circ}$ for all mutation pairs and found that for the V400M:S241T mutation pair, $\sum \Delta G^{\circ}=2.32 \mathrm{kcal} \mathrm{mol}^{-1}$, and for V400M:F1449V mutation 

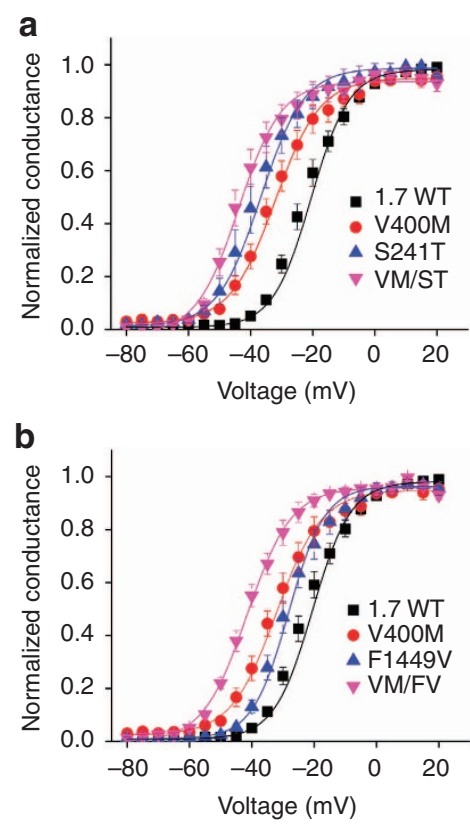

C

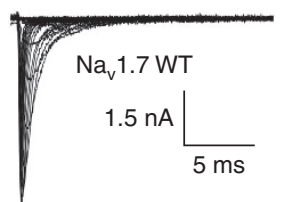

e

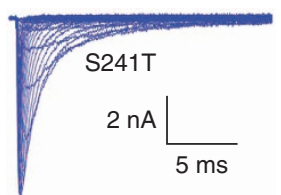

g

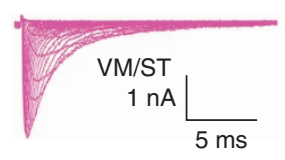

d
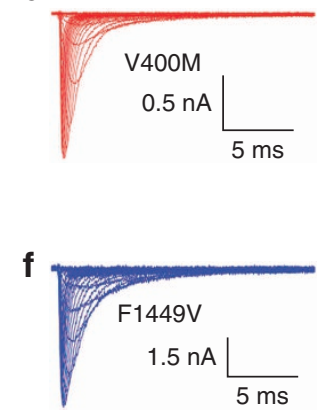

h

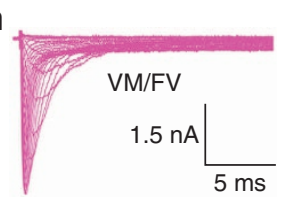

Figure 2 | Mutant cycle analysis of voltage dependence of activation of Nav1.7 mutations. (a) Voltage dependence of activation curves of Nav1.7 WT, V400M, S241T, and V400M/S214T (VM/ST) double mutant channels. Curves were Boltzmann fits of the data. (b) Voltage dependence of activation curves of Nav1.7 WT, V400M, F1449V and V400M/F1449V (VM/FV) double mutant channels. (c-h) Representative traces of current families recorded from HEK293 cells expressing WT (c), V400M (d), S241T (e), F1449V (f), VM/ST double mutant (g) and VM/FV double mutant (h) channels.

pair, the $\sum \Delta G^{\circ}=-0.38 \mathrm{kcal} \mathrm{mol}^{-1}$ (Supplementary Table S1). These data are in good agreement with the fitted curves, and strongly suggest that V400M and S241T are energetically coupled, whereas V400M and F1449V are energetically independent during channel activation.

CBZ depolarizes S241T mutant channel activation. Structural modelling showed atomic proximity between V400 and S241, and mutant cycle analysis demonstrated that S241T is energetically coupled with V400M during activation. Pretreatment of cells with a therapeutic concentration of CBZ $(30 \mu \mathrm{M})$ is known to depolarize activation of V400M but not WT channels ${ }^{9}$. Although residues within S4-S5 linkers of sodium channels have not been implicated in forming the local anaestheticbinding site ${ }^{29}$, we hypothesized that atomic proximity and energetic coupling of V400M and S241T might be paralleled by pharmacological coupling so that they might share pharmacoresponsiveness to CBZ.

To test this hypothesis, the effect of CBZ $(30 \mu \mathrm{M})$ pretreatment, initiated $30 \mathrm{~min}$ before recording 9 , on each mutant's activation was measured in HEK293 cells expressing S241T or F1449V mutant channels. Representative recordings are shown in Fig. 3a-d. Notably, CBZ treatment caused a significant depolarizing shift in the $V_{1 / 2}$ of voltage dependence of activation of S241T mutant channels by $7.1 \mathrm{mV}$ compared with dimethylsulphoxide (DMSO) treatment (DMSO: $-37.6 \pm 1.0 \mathrm{mV}, n=10$; CBZ: $-30.5 \pm$ $1.3 \mathrm{mV}, n=13, P<0.01$, Student's $t$-test, Fig. 3e, Supplementary Table S2). In contrast, CBZ treatment of HEK293 cells expressing F1449V did not show a detectable effect on channel activation compared with DMSO treatment (activation $V_{1 / 2}$ for CBZ treatment: $\quad-27.1 \pm 1.5 \mathrm{mV}, \quad n=8$; activation $V_{1 / 2}$ for DMSO treatment: $-28.8 \pm 1.1 \mathrm{mV}, n=9 ; \quad P>0.05$, Student's $t$-test, Fig. 3f, Supplementary Table S2).

Enhancement of steady-state fast inactivation (a hyperpolarizing shift of steady-state fast inactivation $V_{1 / 2}$ ) is a classic mechanism of action for $\mathrm{CBZ}^{10}$. However, for $\mathrm{Na}_{\mathrm{V}} 1.7$, a therapeutic concentration of $\mathrm{CBZ}$ does not shift steady-state fast inactivation of $\mathrm{WT}$ channel ${ }^{9}$. To determine whether CBZ might have an effect on steady-state fast inactivation of S241T or F1449V mutant channels, HEK293 cells expressing S241T or F1449V mutant channels were treated with DMSO or CBZ $(30 \mu \mathrm{M})$ as described earlier. Steady-state fast inactivation was assessed in response to a 500-ms depolarizing potential. This protocol demonstrated a steady-state fast inactivation $V_{1 / 2}$ for S241T of $-79.9 \pm 1.7 \mathrm{mV}(n=6)$, and a steady-state fast inactivation $V_{1 / 2}$ for $\mathrm{F} 1449$ of $-71.6 \pm 1.5 \mathrm{mV}(n=6)$ with DMSO treatment (Supplementary Table S2), very close to the values found in our previous studies without any drug treatments $^{20,22}$. CBZ $(30 \mu \mathrm{M})$ did not significantly alter the $V_{1 / 2}$ for either S241T or F1449V mutant channels $(-81.5 \pm 2.1 \mathrm{mV}$, $n=6 ;-72.7 \pm 2.2 \mathrm{mV}, n=8$, respectively; $P>0.05$ compared with DMSO treatment, Student's $t$-test, Fig. $3 g, h$ ).

Expression of S241T leads to DRG neuron hyperexcitability. To study the effects of S241T mutant channel on nociceptor excitability, small (20-28 $\mu \mathrm{m}$ diameter) DRG neurons from postnatal day 1-5 rats were isolated and transfected with either human $\mathrm{Na}_{\mathrm{V}} 1.7 \mathrm{WT}$ or S241T mutant channels. Excitability was assessed by current-clamp recording 2 days after transfection. Current threshold, the injection current required to produce a single all-or-none action potential, was determined by applying $200 \mathrm{~ms}$ depolarizing currents of increasing magnitude (5 pA increments). For the neuron transfected with WT channels shown in Fig. 4a, current injection smaller than 225 pA generated small, graded membrane potential depolarization, with the first all-or-none action potential elicited by current injection of $230 \mathrm{pA}$. In contrast, a neuron expressing the S241T mutant channel fired the first action potential in response to current injection of $60 \mathrm{pA}$ (Fig. 4b). On average, expression of S241T mutant caused a significant $\sim 2.7$-fold reduction of action 
a
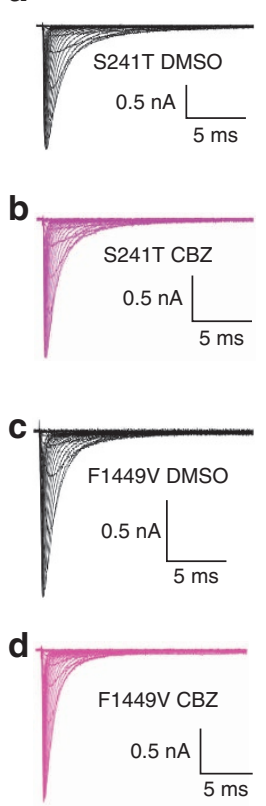

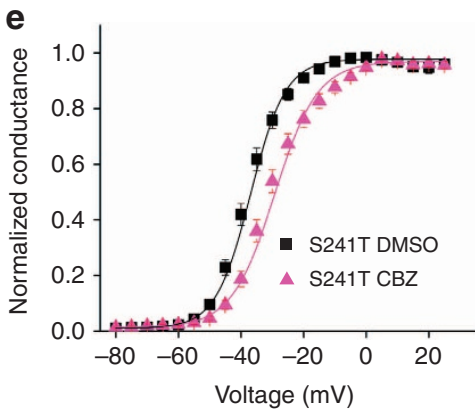

\section{e}

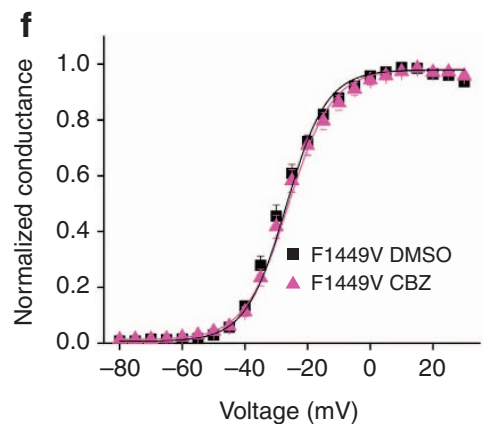

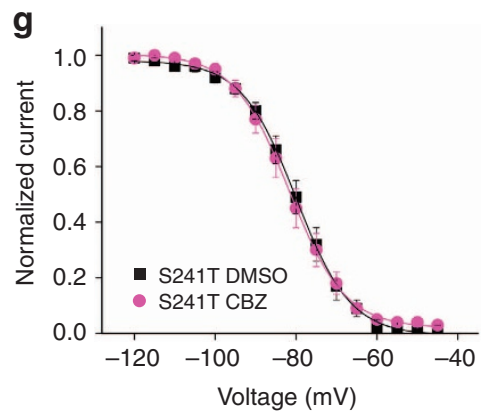

h

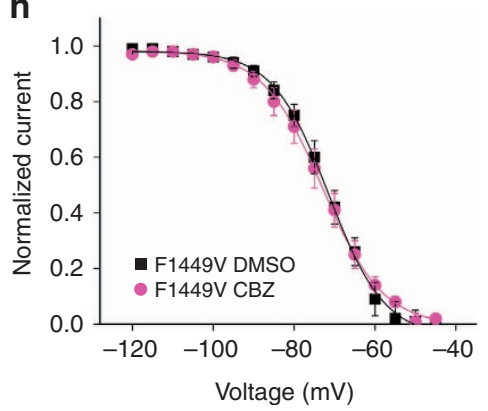

Figure 3 | Voltage dependence of activation and steady-state fast inactivation of S241T and F1449V mutant channels. (a-d) Representative traces of current families recorded from HEK293 cells expressing S241T mutant channel treated with DMSO (a), or with CBZ (b); F1449V mutant channel treated with DMSO (c), or with CBZ (d). (e) The averaged voltage dependence of activation of S241T mutant channel treated with DMSO or CBZ (30 $\mu M$ ) was plotted and fitted with Boltzmann equation. A depolarizing shift of activation of $7.1 \mathrm{mV}$ was observed when S241T mutant channel was treated with CBZ $(P<0.01$, Student's t-test). (f) The averaged voltage dependence of activation of F1449V mutant channel treated with DMSO or CBZ was plotted and fitted with Boltzmann equation. No notable shift in activation curve of F1449V mutant channel was observed. (g-h) The voltage dependence of steady-state fast inactivation in response to 500 ms depolarizing potential for S241T (g) or F1449V (h) mutant channel treated with DMSO or CBZ was plotted and fitted with Boltzmann equation. No notable shift was observed.

potential threshold $(83.5 \pm 18.2 \mathrm{pA}, n=20)$ compared with DRG neurons expressing WT channel $(227.6 \pm 36.7 \mathrm{pA}, \quad n=19$, $P<0.01$, Student's $t$-test, Fig. $4 \mathrm{c}$ ).

The firing frequency of DRG neurons was also assessed by a series of 1 -s current injections from 25 to $500 \mathrm{pA}$ in $25-\mathrm{pA}$ increments. For DRG neurons expressing WT channel, no action potentials or only a single action potential was elicited in response to depolarizing current injection, with an occasional second spike in response to stronger stimuli (Fig. $4 \mathrm{~d}-\mathrm{f}$ ). In contrast, DRG neurons expressing S241T fired repetitively in response to low and high current injections (Fig. 4g-i). To compare firing frequency of DRG neurons expressing WT or S241T channels, the number of spikes elicited by 1-s current injection at various stimulus intensities was averaged (Fig. 4j). DRG neurons expressing S241T mutant channel fired significantly more spikes than DRG neurons expressing WT channel at all stimulus intensities $\geq 125$ pA (Fig. 4j, $P<0.05$, Mann-Whitney test). Resting membrane potential (RMP) is known to affect DRG neuron excitability $^{30}$. We therefore also assessed RMP of DRG neurons expressing WT or S241T mutant channels but did not observe a significant difference in RMPs (Fig. 4k). Taken together, these data show that expression of S241T mutant channels induces DRG neuron hyperexcitability.

CBZ normalizes excitability of DRG neurons expressing S241T. To test the prediction from our structural modelling and mutant cycle analysis, we assessed the effect of CBZ on hyperexcitability of DRG neurons expressing S241T or F1449V mutant channels using current-clamp recording. DRG neurons were treated with either DMSO or CBZ $(30 \mu \mathrm{M})$ initiated $30 \mathrm{~min}$ before recording as mentioned above.

Current threshold was assessed first: the response of a representative neuron expressing S241T treated with DMSO is shown in Fig. 5a. Current threshold for this neuron was $75 \mathrm{pA}$. In contrast, current threshold of a representative neuron expressing S241T treated with CBZ is shown in Fig. 5b. Current threshold for this neuron is $170 \mathrm{pA}$. On average, CBZ treatment resulted in a significant $\sim$ two fold increase in current threshold for DRG neurons expressing S241T mutant channel (CBZ treatment: $162.7 \pm 24.4 \mathrm{pA}, n=28$; DMSO treatment: $90.4 \pm 13.2 \mathrm{pA}, n=27$, $P<0.01$, Student's $t$-test; Fig. $5 \mathrm{c}$ ), indicating that CBZ treatment is likely to attenuate the excitability of DRG neurons expressing S241T mutant channel.

Our previous studies demonstrated that expression of F1449V mutant channel in DRG neurons reduces the current threshold and increases the firing frequency in response to sustained depolarizing stimuli ${ }^{21}$. As CBZ did not produce a depolarizing shift in F1449V mutant channel activation curve (Fig. 3f), we hypothesized that CBZ would not normalize firing properties of DRG neurons expressing F1449V mutant channel. Again, we assessed the current threshold first: as illustrated in Fig. 5d,e, current threshold of DRG neurons expressing F1449V mutant channels was similar between CBZ and DMSO treatments. For the population of neurons in these experiments, no significant difference was found for current threshold (DMSO, $153.5 \pm 17.9 \mathrm{pA}, n=29 ; \mathrm{CBZ}, 165.5 \pm 19.7 \mathrm{pA}, n=28, P>0.05$, Student's $t$-test, Fig. 5f).

We further tested the effect of CBZ treatment on the firing frequency of DRG neurons expressing either the S241T or 

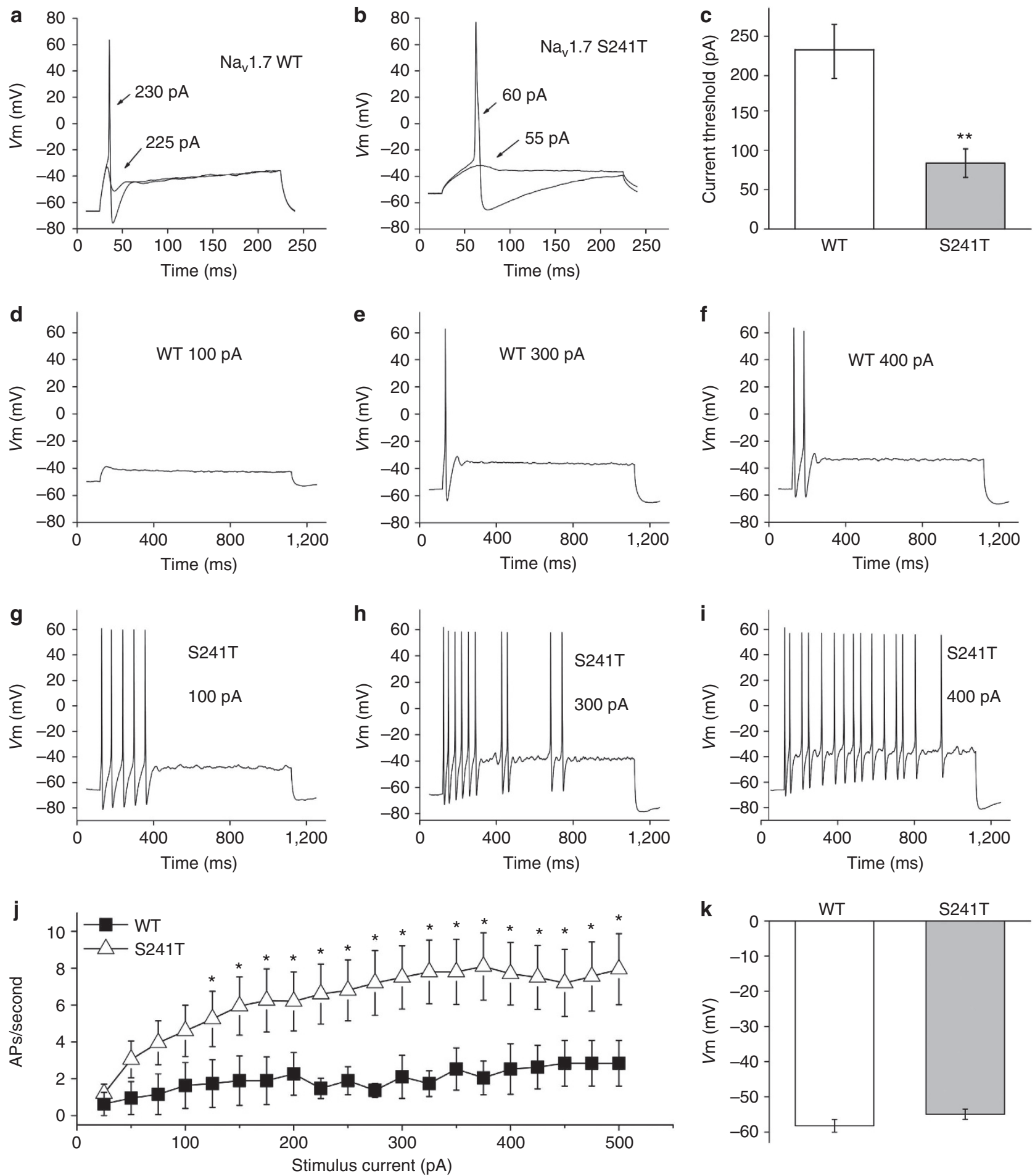

Figure 4 | Current-clamp analysis of DRG neurons expressing WT or S241T mutant channel. (a) Representative DRG neuron expressing Nav1.7 WT channel showed sub-threshold response to $225 \mathrm{pA}$ current injection and subsequent action potential evoked by injection of $230 \mathrm{pA}$, which was the current threshold for this neuron. (b) Representative DRG neuron expressing Nav1.7-S241T mutant channel showed sub-threshold response to 55 pA current injection and subsequent action potential evoked by injection of $60 \mathrm{pA}$. (c) Comparison of current threshold for DRG neurons expressing WT and S241T mutant channels. Expression of S241T channel reduced current threshold significantly ( ${ }^{\star \star} P<0.01$, Student's $t$-test). Current threshold for WT: $(227.6 \pm 36.7 \mathrm{pA}, n=19)$; for S241T: $(83.5 \pm 18.2 \mathrm{pA}, n=20)$. ( $\mathbf{d}-\mathbf{f})$ Responses of a representative DRG neuron expressing WT channel to 1-s-long depolarizing current steps at 100 (d), 300 (e) and 400 (f) pA current injection. (g-i) Responses of a representative DRG neuron expressing S241T mutant channel to 1-s-long depolarizing current steps at $100(\mathbf{g}), 300(\mathbf{h})$ and $400(\mathbf{i})$ pA current injection. The difference in responses is apparent across this range. (j) The averaged number of action potentials between DRG neurons expressing WT and S241T mutant channel was compared. The response of DRG neurons expressing WT channel to current injection was significantly different compared with DRG neurons expressing S241T mutant channel across a range (125-500 pA) of step current injections ( ${ }^{\star} P<0.05$, Mann-Whitney test). (k) Averaged resting membrane potentials for DRG neurons expressing WT or $\mathbf{S 2 4 1 T}$ mutant channel were not statistically different. Results are presented as mean \pm s.e.m. 

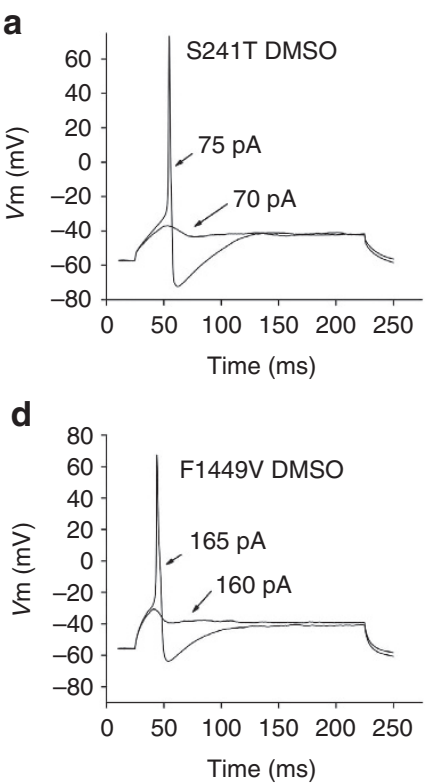
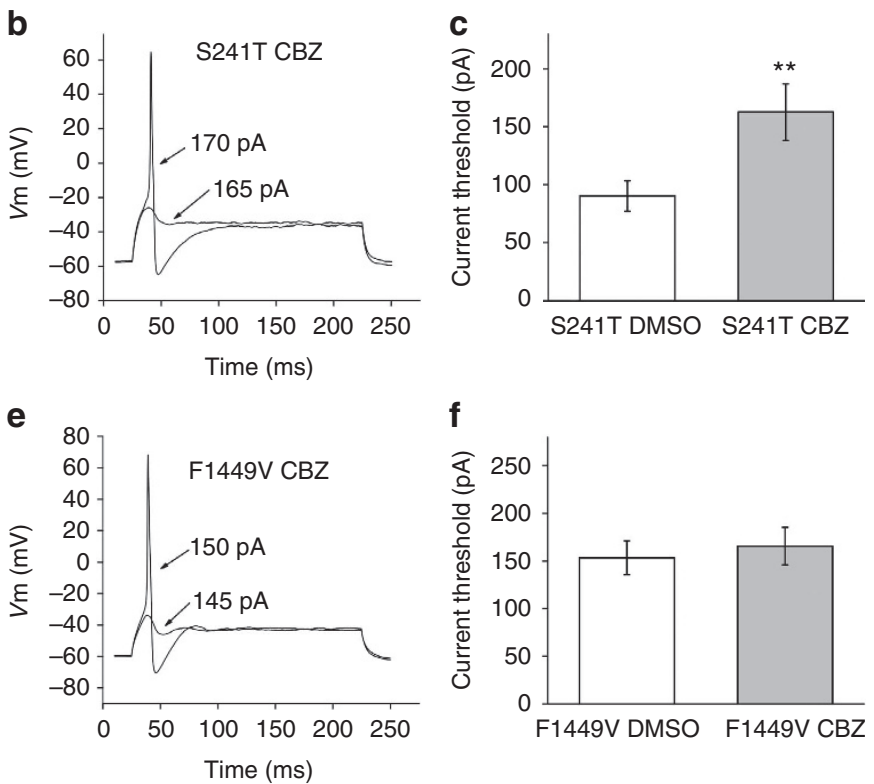

Figure 5 | Current thresholds of CBZ- or DMSO-treated DRG neurons expressing S241T or F1449V mutant channels. (a,b) Sub- and supra-threshold responses of representative DRG neurons expressing S241T mutant channel treated with DMSO (a) or $30 \mu \mathrm{M}$ CBZ (b) are shown. (c) Comparison of current threshold for DRG neurons expressing S241T mutant channel treated with DMSO or $30 \mu \mathrm{M} \mathrm{CBZ.} \mathrm{CBZ} \mathrm{treatment} \mathrm{increased} \mathrm{the} \mathrm{current} \mathrm{threshold}$ significantly ( ${ }^{\star} P<0.01$, Student's $t$-test). Current threshold for DMSO-treated DRG neurons: $90.4 \pm 13.2 \mathrm{pA}(n=27)$; for CBZ-treated DRG neurons: $162.7 \pm 24.4 \mathrm{pA}(n=28)$. (d,e) Sub- and supra-threshold responses of DRG neurons expressing F1449V mutant channel treated with DMSO (d) or $30 \mu \mathrm{M} \mathrm{CBZ}$ (e) are shown. (f) Comparison of current threshold for DRG neurons expressing F1449V mutant channel with the treatment of DMSO $(153.5 \pm 17.9 \mathrm{pA}, n=29)$ or $30 \mu \mathrm{M} \mathrm{CBZ}(165.5 \pm 19.7 \mathrm{pA}, n=28)$. No significant difference was found $(P>0.05$, Student's $t$-test). Results are presented as mean \pm s.e.m.

F1449V mutant channels. Robust repetitive firing was seen for neurons expressing S241T mutant channel treated with DMSO (Fig. 6a-c). In contrast, pretreatment with CBZ dramatically reduced the number of action potentials in DRG neurons expressing S241T mutant channel (Fig. 6d-f), with a statistically significant reduction of firing frequency at all stimulus intensities $\geq 100$ pA $(P<0.05$, Mann-Whitney test, Fig. $6 \mathrm{~g})$. This effect was not confounded by changes in RMPs, as CBZ did not affect RMPs of DRG neurons expressing mutant channels (Figs $6 \mathrm{~h}$ and $7 \mathrm{~h}$ ). Taken together, our data demonstrate that CBZ attenuates the hyperexcitability of DRG neurons expressing S241T mutant channel.

In contrast, CBZ did not attenuate the abnormal repetitive firing of DRG neurons expressing F1449V mutant channel. Repetitive firing was observed for neurons expressing F1449V mutant channel treated with DMSO (Fig. $7 \mathrm{a}-\mathrm{c}$ ) as well as treated with $\mathrm{CBZ}$ (Fig. $7 \mathrm{~d}-\mathrm{f}$ ). Across a range of current injections, the firing frequency for CBZ- and DMSO-treated neurons was comparable in response to current injection $\leq 200$ pA (Fig. $7 \mathrm{~g}$ ). Although firing frequency started to diverge with stronger injection, the effect of CBZ did not reach statistical significance even at current injection of $500 \mathrm{pA}$ (Fig. $7 \mathrm{~g}$ ). Together with our results on V400M and S241T mutant channels, these data suggest that the effect of CBZ is mutant specific and agrees well with our predication from structural modelling and mutant cycle analysis.

\section{Discussion}

Using structural modelling, based upon the recently solved crystal structure of a bacterial voltage-gated sodium channel ${ }^{13}$, we report that S241 within the DI/S4-S5 linker, while 159 amino acids distant from V400 in the channel peptide, is located close to V400 in the folded structure of the human $\mathrm{Na}_{\mathrm{V}} 1.7$ sodium channel.
Using mutant cycle analysis, we have demonstrated that $\mathrm{Na}_{\mathrm{V}} 1.7-$ V400M and -S241T mutant channels are energetically coupled during channel activation, suggesting that they contribute to the same feature of activation. As V400M mutation responds favourably to CBZ by shifting activation of $\mathrm{V} 400 \mathrm{M}^{9}$, we reasoned that energetic coupling of V400M and S241T during channel activation might be paralleled by pharmacological coupling. Using voltage-clamp recording, we found that a therapeutic concentration of CBZ indeed normalizes the activation of S241T mutant channel. Moreover, we further show that this normalization of activation is accompanied by increased current threshold and reduced firing frequency of DRG neurons expressing S241T mutant channel. In contrast, V400M and F1449V mutants were not energetically coupled, and CBZ had no detectable effect on activation of F1449V or on the firing properties of DRG neurons expressing F1449V mutant channel.

Human and rodent studies have demonstrated that the $\mathrm{Na}_{\mathrm{V}} 1.7$ channel is essential for pain signalling and have validated this channel as a promising drug target for the development of novel pain therapeutics ${ }^{4}$. Structural and functional studies of $\mathrm{Na}_{\mathrm{V}} 1.7$ mutant channels may provide insight into understanding mechanistic features of channel gating and its interaction with drugs. Previous studies of sodium channel structure and function were largely based on information obtained from the crystal structure of potassium channels ${ }^{22,25,31}$. Availability of the crystal structure of a bacterial voltage-gated sodium channel $\mathrm{NavAb}^{13}$ permitted construction of a more realistic mammalian voltagegated sodium channel model. It is worth noting that bacterial $\mathrm{Na}_{\mathrm{V}}$ channels are four-fold symmetric, assembled from identical subunits, whereas mammalian $\mathrm{Na}_{\mathrm{V}}$ channels have four nonidentical domains encoded by a single protein. Alignment of $\mathrm{NavAb}$ and the four transmembrane domains of the $\mathrm{Na}_{\mathrm{V}} 1.7$ channel is shown in Supplementary Table S3 and superposition of 

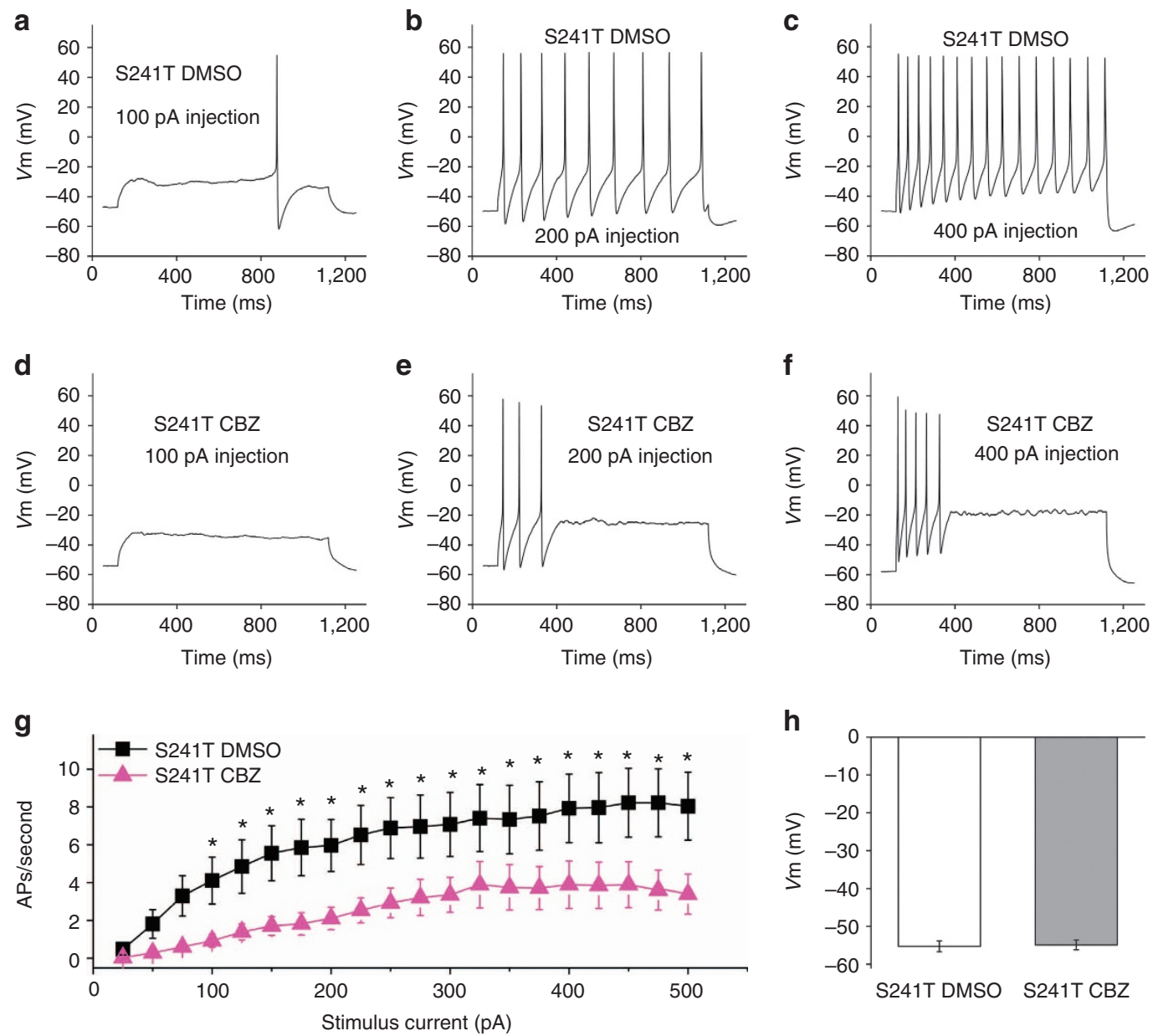

Figure 6 | Firing frequencies and membrane potentials of CBZ- or DMSO-treated DRG neurons expressing S241T. (a-c) Responses of a representative DRG neuron expressing S241T mutant channel treated with DMSO to 1s long depolarization current steps at 100 (a), 200 (b) and 400 (c) pA current injection. (d-f) Similar recordings from a representative DRG neuron expressing S241T mutant channel treated with $30 \mu \mathrm{M}$ CBZ at 100 (d), 200 (e), and 400 (f) pA current injection. (g) Averaged response for DRG neurons expressing S241T mutant channel treated with DMSO $(n=27)$ or CBZ $(n=28)$ are summarized. CBZ statistically reduced firing frequency starting from $100 \mathrm{pA}$ current injection $\left({ }^{\star} P<0.05\right.$, Mann-Whitney test). (h) Averaged RMP between DRG neurons expressing S241T mutant channel treated with DMSO or CBZ were not statistically different (DMSO: $-55.3 \pm 1.4 \mathrm{mV}, n=27 ; \mathrm{CBZ}$ :

$-54.9 \pm 1.3 \mathrm{mV}, n=28, P>0.05$, Student's $t$-test). Results are presented as mean \pm s.e.m.

the transmembrane domains of $\mathrm{Na}_{\mathrm{V}} 1.7$ with $\mathrm{NavAb}$ is shown in Fig. 8. Despite the difference outlined above, the bacterial $\mathrm{Na}_{\mathrm{V}}$ channel is the closest template for studying mammalian $\mathrm{Na}_{\mathrm{V}}$ channel. Additionally, recent enhancements to I-TASSER $^{17}$, a protein prediction package that has been successfully used to predict structure of many proteins including potassium channels for functional studies ${ }^{32,33}$, have provided improved predictions of membrane-bound proteins structure ${ }^{15,34}$. This method is not a simple homology modelling tool because the threading alignments from templates have been broken into pieces, which are used to reassemble the global structure of the membrane domains under the guide of a composite physics- and knowledgebased force field. As a result, it has been systematically demonstrated in community-wide CASP experiments that GPCR-ITASSER models can be significantly closer to the target structure than the homologous modelling ${ }^{35}$. Taking advantage of these new developments, we constructed a structural model for the human $\mathrm{Na}_{\mathrm{V}} 1.7$ channel and used it in this study to investigate the participation of different residues in channel activation, and to make predictions about drug-channel interaction.

We used thermodynamic mutant cycle analysis, a wellestablished tool, to quantitatively assess the energetic independence or coupling of two mutations in affecting channel activation. Mutant cycle analysis has been used to understand transition of pore elements during potassium channel gating ${ }^{23}$, interactions between ion channels and toxins ${ }^{24,28}$ and identification of ion-pairforming residues of the voltage-sensor domain in a bacterial sodium channel $(\mathrm{NaChBac})$ during voltage-gated activation ${ }^{25-27}$. We show here that mutant cycle analysis can be used to test the hypothesis that different pathogenic IEM mutations (S241T, V400M and F1449V), all of which cause a hyperpolarizing shift of channel activation, participate in different steps of channel gating and have distinct pharmacoresponsiveness. More importantly, this analysis allowed us to predict that S241T mutant channel may interact with CBZ in a similar fashion as V400M mutant channel owing to their energetic coupling for channel activation.

Our structural model revealed that the V400 side chain points towards the DI/S4-S5 linker and is atomically close to and facing the S241 residue located in this linker, suggesting that mutations of these two residues might affect the same step in channel activation. The interaction between S4-S5 linker and S6 during channel gating has been suggested in voltage-gated $\mathrm{K}^{+}\left(\mathrm{K}_{\mathrm{V}}\right)$ channel $^{36-38}$. More recently, an all-atom molecular dynamics 

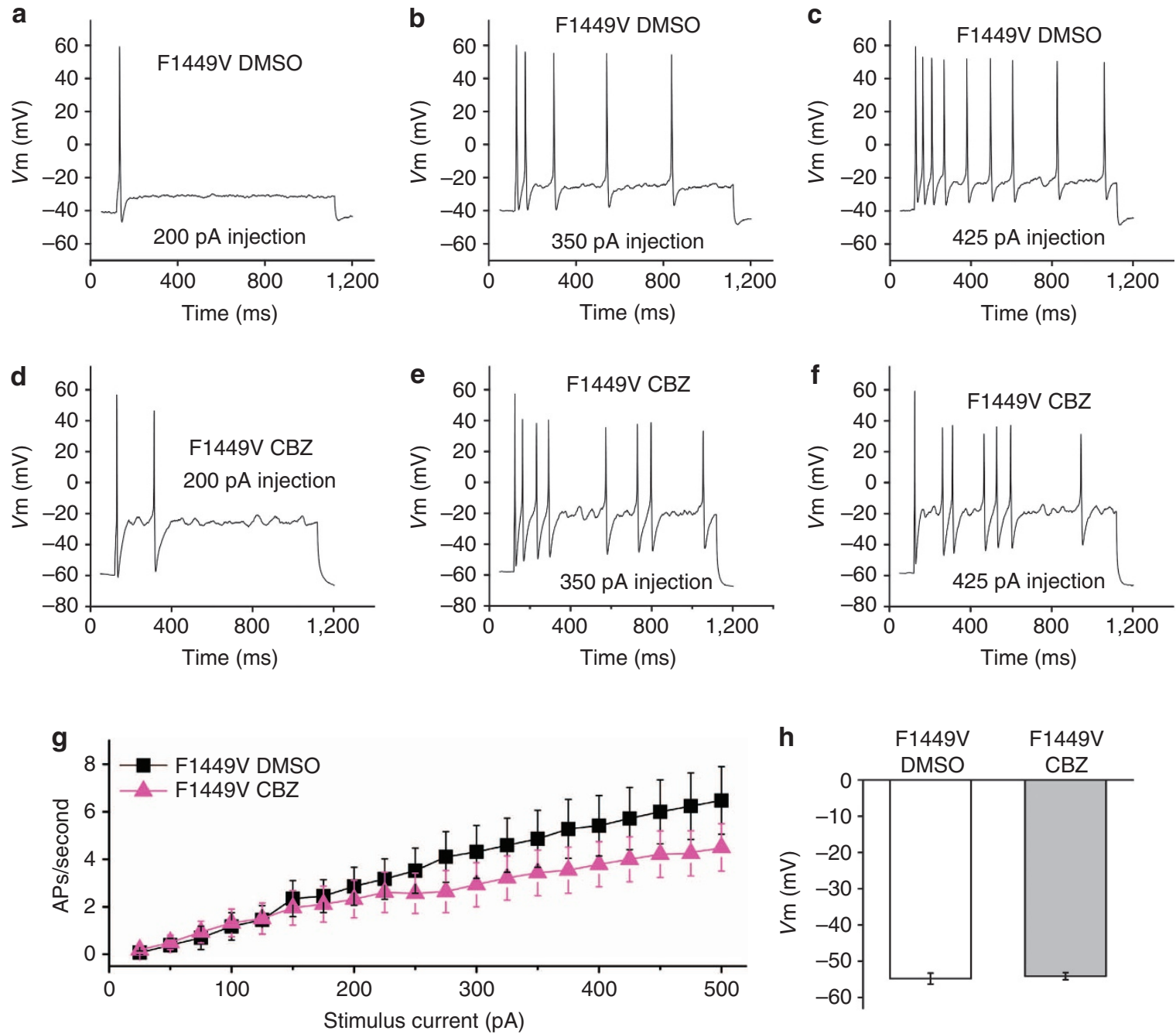

Figure 7 | Firing frequencies and membrane potentials of CBZ- or DMSO-treated DRG neurons expressing F1449V. (a-c) Responses of a representative DRG neurons expressing F1449V mutant channel treated with DMSO to 1-s-long depolarization current steps at 200 (a), 350 (b) and 425 (c) pA current injection. (d-f) Similar recordings from a representative DRG neuron expressing F1449V mutant channel treated with CBZ at 200 (d), 350 (e) and 425 (f) pA current injection. (g) Averaged firing frequencies for DRG neurons expressing F1449V mutant channel treated with DMSO ( $n=29)$ or CBZ ( $n=28$ ) were compared and no statistical difference was found across the entire range ( $P>0.05$, Mann-Whitney test). (h) Averaged RMPs between DRG neurons expressing F1449V mutant channel treated with DMSO or CBZ were not statistically different (DMSO: $-54.8 \pm 1.5 \mathrm{mV}, n=29 ; \mathrm{CBZ}:-54.1 \pm 1.0 \mathrm{mV}$, $n=28, P>0.05$, Student's $t$-test). Results are presented as mean \pm s.e.m.

study of $\mathrm{K}_{\mathrm{V}}$ channel gating mechanism has extended these findings by showing that channel activation imposes a pulling force on S4-S5 linker, which perturbs the interaction between S4S5 linker and S6, leading to channel opening ${ }^{39}$. Coupling of poreforming helix to voltage sensors is also seen in $\mathrm{Na}_{\mathrm{V}}$ channels ${ }^{40,41}$, most likely through S4-S5 linker ${ }^{42}$. A residue at the beginning of the S4-S5 linker is likely to form an interacting pair with residue at the tail end of the $\mathrm{S} 6$ helix $^{42}$, further supporting the potential interaction of S4-S5 linker and S6 helix. On the other hand, F1449V may affect different steps in activation: the $\mathrm{Na}_{\mathrm{V}} 1.7$ structural model places F1449 at the cytoplasmic tip of the S6 helix, suggesting that F1449 contributes to a hydrophobic ring at the cytoplasmic vestibule of the pore, consists of aromatic residues at equivalent positions in the S6 from four domains, acting as the activation gate of the channel. Taken together, our results suggest that V400M or S241T mutation may disrupt the tight packing of S4-S5 linker with S6 pore-forming helix, affecting channel activation; whereas the F1449V mutation may disrupt the hydrophobic ring and destabilize the channel pre- open state, hyperpolarizing activation via a different structural action $^{21,22}$.

Mutational analysis and functional studies have identified residues within S6 segments of domains III and IV to be important in forming drug-binding site(s) for local anaesthetic and antiepileptic drugs, including CBZ (see refs 43-45 for review). The binding of a few sodium channel blockers (for example, local anaesthetic drugs, anticonvulsant drugs) to the pore of voltage-gated sodium channels has been studied using structural modeling ${ }^{29,46}$. CBZ has been shown to interact with three key residues of $\mathrm{Na}_{\mathrm{V}} 1.2$ channel (Leu1465 of DIII-S6, Phe1764 of DIV-S6 and Tyr1771 of DIV-S6) ${ }^{29}$. In addition, N434, located near the middle of the DI/S6 helix, is also important for drug binding of $\mathrm{Na}_{\mathrm{V}} 1.4$ (ref. 47). IEM mutation $\mathrm{N} 395 \mathrm{~K}$ of $\mathrm{Na}_{\mathrm{V}} 1.7$ channel, which corresponds to $\mathrm{N} 434$ in $\mathrm{Na}_{\mathrm{V}} 1.4$, is suggested to be located within the local anaesthetic-binding site of $\mathrm{Na}_{\mathrm{V}} 1.7$. N395K mutation has been shown to depolarize activation of $\mathrm{Na}_{V} 1.7$ as well as significantly reduce use-dependent inhibition by lidocain $\mathrm{e}^{11}$. In addition, it has been found that the binding of local 

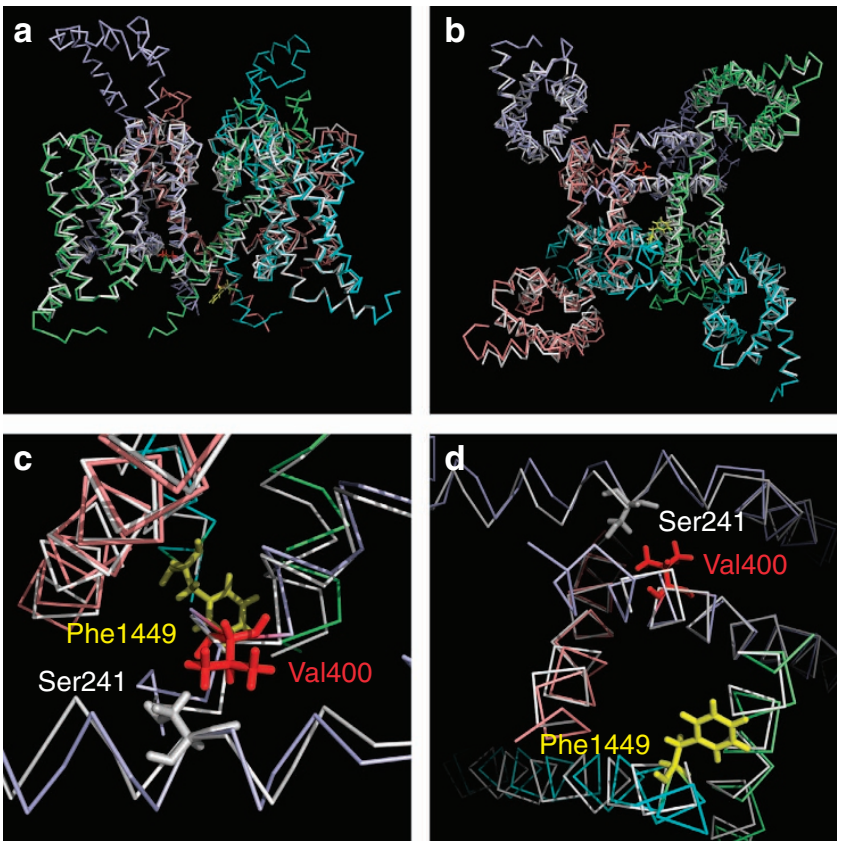

Figure 8 | Alignment of Nav1.7 structural model with NavAb structure.

(a) Intra-membrane view of structural model of Nav1.7 channel

transmembrane domains aligned with NavAb structure (3RVY). Nav1.7

Domain I, lightblue; Domain II, salmon; Domain III, cyan; Domain IV, lime. $\mathrm{NavAb}$, white. (b) Cytosolic view of the structural model of Nav1.7 channel transmembrane domains aligned with NavAb structure. (c) Close-up intramembrane side view of the area containing S241 (grey), V400 (red) and F1449 (yellow) residues. (d) Close-up cytosolic view of the area containing S241, V400 and F1449 residues.

anaesthetics (for example, lidocaine) to $\mathrm{Na}_{\mathrm{V}}$ channel may lead to allosteric coupling of the binding site(s) of pore-forming helix to voltage sensors, especially in domain III and $\mathrm{IV}^{48}$. It is further suggested that S4-S5 linker and the intracellular end of S5 and S6 may be critical in coupling S6 helix to voltage-sensor movement in the presence of local anaesthetics ${ }^{49}$.

These results imply that the effects of CBZ on $\mathrm{Na}_{\mathrm{V}} 1.7-\mathrm{S} 241 \mathrm{~T}$ and -V400M mutant channels are not likely to be attributable to direct effects of these mutations on the drug-binding site(s). Alternatively, our data may be interpreted as suggesting a role for CBZ as a chemical chaperone for S241T and V400M mutant channels, rather than as a channel blocker. According to this hypothesis, CBZ-channel interaction may lead to stabilization of the S241T and V400M mutant channel in a WT-like conformation, and cause a depolarizing shift in activation, a biophysical change that contributes to attenuation of hyperexcitability of DRG neurons expressing mutant channels.

In summary, our results demonstrate that structural modelling and mutant cycle analysis can reveal the effect of sodium channel mutations on channel gating, and predict the responses of sodium channel mutations to a pharmacotherapeutic agent. We suggest that a similar approach, initially screening channels of interest for amino-acid variants that enhance pharmacoresponsiveness to existing agents and then using these variants as 'seeds' for further identification of other variants that enhance pharmacosensitivity, may permit identification of genomically defined subgroups of the population that are pharmacoresponsive.

\section{Methods}

Structural modelling. Mammalian $\mathrm{Na}_{\mathrm{V}} 1.7$ sodium channel is made up of four transmembrane domains, named I, II, III, IV linked by cytoplasmic loops. The transmembrane helix structural model of human $\mathrm{Na}_{\mathrm{V}} 1.7$ sodium channel was constructed in two steps. First, each of the four transmembrane domains was modelled separately using an advanced membrane-bound protein prediction algorithm GPCR-ITASSER ${ }^{16,50,51}$. Then, the whole channel model was built by structurally aligning four individual domain models to a global structural template of the recently solved bacterial sodium channel

The GPCR-ITASSER algorithm is an advanced algorithm for transmembrane helical structure modelling. The program first uses a multiple threading procedure LOMETS $^{14}$ to identify the putative-related template structures in the PDB. The structural fragments (mainly $\alpha$-helices) are then excised from the threading templates and assembled into full-length models by replica-exchange Monte Carlo simulations. In addition to the inherent I-TASSER force field, five transmembraneprotein-specific energy terms were also used to describe the interactions between transmembrane domain and membrane. (1) Membrane-repulsive energy is introduced for reducing the clash between intra- and extracellular domain and bilayer membrane. (2) Extracellular hydrophilic interactions for the hydrophilic interactions for residues inside and outside membrane. (3) Hydrophobic moment energy for the hydrophobic interaction between transmembrane helix and membrane. (4) Aromatic interactions for enhancing the specific interactions between aromatic-aromatic residues. (5) Cation $-\pi$ interactions for specific noncovalent-binding propensities between TM helices. Finally, models of the lowest free energy are identified by clustering the simulation trajectories using SPICER $^{52}$ and are refined at the atomic level by a fragment-guided dynamic simulation program FG-MD ${ }^{50}$

We assembled the four transmembrane domains models in a clockwise order viewed from extracellular side as suggested by previous literature ${ }^{53,54}$ Each single domain model was aligned to the corresponding domain of the recently solved bacterial sodium channel by the structural alignment algorithm $\mathrm{TM}_{\text {-align }}{ }^{55}$. The resultant four domain complete structural model was refined again by FG-MD ${ }^{50}$ to accommodate inter-domain steric clashes and improve the model quality.

Plasmid preparation and HEK293 cell transfection. TTX-resistant human $\mathrm{Na}_{\mathrm{V}} 1.7$ wild-type (WT) channel $\left(\mathrm{hNa}_{\mathrm{V}} 1.7 \mathrm{r}\right)$ was created based on the hNa 1.7 (GenBank accession codes: NM_002977.3 (mRNA); NP_002968.1 (protein). All other tested mutations were constructed on $\mathrm{hNa} / \mathrm{V} 1.7 \mathrm{r}$ background using Quick Change XL site-directed mutagenesis kit (Stratagene, La Jolla, CA, USA). WT or the mutant channels were transfected into HEK293 cells together with human $\beta-1$ and $\beta-2$ subunit $^{56}$ using Lipofectamine reagent (Invitrogen, Carlsbad, CA, USA). HEK293 cells were maintained in 1:1 Dulbecco's modified Eagle's media (DMEM)/F12 supplemented with 10\% fetal bovine serum (FBS, Hyclone) in a humidified $5 \% \mathrm{CO}_{2}$ incubator at $37^{\circ} \mathrm{C}$. HEK293 cells were seeded onto poly-l-lysine-coated glass coverslips (BD Biosciences, San Jose, CA, USA) in a 24 -well plate 1 day before transfection. Recording was performed 1 day after transfection.

Voltage-clamp recording of sodium channel in HEK cells. Whole-cell voltageclamp recordings were performed using the following solutions: the extracellular solution contained the following (in $\mathrm{mM}$ ): $140 \mathrm{NaCl}, 3 \mathrm{KCl}, 1 \mathrm{MgCl}_{2}, 1 \mathrm{CaCl}_{2}$, 20 Dextrose and 10 HEPES, $\mathrm{pH}=7.3$ with $\mathrm{NaOH}(320 \mathrm{mOsm}$ adjusted with dextrose). The pipette solution contained the following (in $\mathrm{mM}$ ): $140 \mathrm{Cs}$-Fluoride, $10 \mathrm{NaCl}, 1.1$ EGTA, 10 HEPES, 20 Dextrose, $\mathrm{pH}=7.3$ with $\mathrm{CsOH}(310 \mathrm{mOsm}$ adjusted with dextrose). Patch pipettes had a resistance of 1-2 $\mathrm{m} \Omega$ when filled with pipette solution. After achieving whole-cell recording configuration, the pipette and cell capacitance were manually minimized using the Axopatch 200B (Molecular Devices) compensation circuitry. Series resistance and prediction compensation (80-90\%) was applied to reduce voltage errors. The recorded currents were digitized using pClamp software and a Digidata 1440A interface (Molecular Devices) at a rate of $50 \mathrm{kHz}$ after passing through a low-pass Bessel filter setting of $10 \mathrm{kHz}$. Linear leak and residual capacitance artifacts were subtracted out using a $\mathrm{P} / \mathrm{N}$ method provided with the Clampex software. Recording was initiated after a 5-min equilibration period after breaking in whole-cell configuration. CBZ was purchased from Sigma, dissolved in DMSO. For CBZ experiments, cells were treated with either CBZ $(30 \mu \mathrm{M})$ or DMSO in incomplete medium without FBS for $30 \mathrm{~min}$ before recording and the CBZ or DMSO was maintained in the bath solution during the recording as described previously ${ }^{9}$

Data analysis was performed using Clampfit (Molecular Devices) and Origin (Microcal Software). To generate activation curves, cells were held at $-120 \mathrm{mV}$ and stepped to potentials of -80 to $+40 \mathrm{mV}$ in $5-\mathrm{mV}$ increments for $100 \mathrm{~ms}$. Peak inward currents were automatically extracted by Origin and fitted with BoltzIV function to determine the voltage at half activation $\left(V_{1 / 2}\right)$, activation curve slope at half activation $(Z)$ and reversal potential $\left(E_{\mathrm{Na}}\right)$ for each recording. Conductance was calculated as $G=I /\left(V \mathrm{~m}-E_{\mathrm{Na}}\right)$ and normalized by the maximum conductance value and fit with Boltzmann equation. To generate steady-state fast-inactivation curves, cells were stepped to inactivating potentials from -120 to $-40 \mathrm{mV}$ for $500 \mathrm{~ms}$ followed by a 40 -ms step to $-10 \mathrm{mV}$ as described previously ${ }^{21,57}$. Peak inward currents obtained from steady-state fast inactivation were normalized by maximum current amplitude and fitted with a Boltzmann equation. 
Mutant cycle analysis. Double mutations (VM/ST and VM/FV) were created using site-directed mutagenesis. A total of six constructs (WT, V400M, S241T, F1449V, VM/ST, VM/FV) were expressed in HEK293 cells separately for patchclamp analysis. The voltage dependence of activation for these mutations was analysed and a $G-V$ curve was generated and fitted with Boltzmann equation to obtain $V_{1 / 2}$ and $Z$. Using $V_{1 / 2}$ and $Z$, the free energy to switch the channel from closed to the open state was calculated as $\Delta G^{\circ}(\mathrm{C} \rightarrow \mathrm{O})(\mathrm{kcal} / \mathrm{mol})=-F Z V_{1 / 2}$. The additional free energy required for the mutant channel to open relative to the WT channel was calculated as $\Delta \Delta G^{\circ}=\Delta\left(F Z V_{1 / 2}\right)=-F\left(Z_{\mathrm{mut}} V_{1 / 2 \mathrm{mut}}-Z_{\mathrm{wt}} V_{1 / 2 \mathrm{wt}}\right)$. Magnitude of non-additivity (or the non-additive coupling free energy) was calculated as $\Sigma \Delta G^{\circ}=\Delta \Delta\left(F Z V_{1 / 2}\right)=-F\left[\left(Z_{\mathrm{wt}} V_{1 / 2 \mathrm{wt}}-Z_{\text {mut } 1} V_{1 / 2 \text { mut1 }}\right)-\left(Z_{\text {mut } 2} V_{1 / 2 \text { mut } 2}-\right.\right.$ $Z_{\text {mut1,mut } 2} V_{1 / 2}$ mut1,mut2)] (refs 23-28).

Isolation and transfection of DRG neurons. DRG of Sprague-Dawley rat pups (postnatal day 1-5) were isolated and cultured as described previously ${ }^{58,59}$ Dissected ganglia were placed in ice-cold oxygenated complete saline solution (CSS), which contained the following (in $\mathrm{mM}$ ): $137 \mathrm{NaCl}, 5.3 \mathrm{KCl}, 1 \mathrm{MgCl}_{2}$, 25 sorbitol, $3 \mathrm{CaCl}_{2}$ and $10 \mathrm{HEPES}, \mathrm{pH}$ 7.2. DRGs were then transferred to oxygenated $37^{\circ} \mathrm{C}$ CSS solution containing $1.5 \mathrm{mg} \mathrm{ml}^{-1}$ collagenase A (Roche Applied Science) and $0.6 \mathrm{mM}$ EDTA and incubated with gentle agitation at $37^{\circ} \mathrm{C}$ for $20 \mathrm{~min}$. This solution was exchanged with oxygenated, $37^{\circ} \mathrm{C}$ CSS solution containing $1.5 \mathrm{mg} \mathrm{ml}^{-1}$ collagenase D (Roche Applied Science), $0.6 \mathrm{mM}$ EDTA and $30 \mathrm{U} \mathrm{ml}^{-1}$ papain (Worthington Biochemicals) and incubated with gentle agitation at $37^{\circ} \mathrm{C}$ for $20 \mathrm{~min}$. The solution was then aspirated and ganglia were triturated in DRG media: DMEM/F12 (1:1) with $100 \mu \mathrm{ml}^{-1}$ penicillin, $0.1 \mathrm{mg} \mathrm{ml}^{-1}$ streptomycin (Invitrogen), and 10\% FBS, which contained $1.5 \mathrm{mg} \mathrm{ml}^{-1}$ bovine serum albumin (Sigma-Aldrich) and $1.5 \mathrm{mg} \mathrm{ml}^{-1}$ trypsin inhibitor (Roche Applied Science).

WT, S241T or F1449V mutant channels were transiently transfected into the DRG neurons, respectively, along with enhanced green fluorescent protein (GFP), by electroporation with a Nucleofector II (Amaxa) system using basic Neuron SCN Nucleofector and program ' $\mathrm{SCN}$ basic program 6'. The ratio of sodium channel to GFP constructs was 10:1. Transfected neurons were allowed to recover for $5 \mathrm{~min}$ at $37^{\circ} \mathrm{C}$ in $0.5 \mathrm{ml}$ of $\mathrm{Ca}^{+}+$-free DMEM. The cell suspension was then diluted with DRG media containing $1.5 \mathrm{mg} \mathrm{ml}^{-1}$ bovine serum albumin and $1.5 \mathrm{mg} \mathrm{ml}^{-1}$ trypsin inhibitor, $80 \mu \mathrm{l}$ mixture was placed on $12 \mathrm{~mm}$ circular poly-d-lysine/ laminin-precoated coverslips (BD biosciences), and the cells were incubated at $37^{\circ} \mathrm{C}$ in $5 \% \mathrm{CO}_{2}$ for $30 \mathrm{~min}$. DRG media ( $1 \mathrm{ml}$ per well), supplemented with $50 \mathrm{ng} \mathrm{ml}^{-1}$ each of mouse NGF (Alomone Labs) and glial cell line-derived neurotrophic factor (GDNF, Peprotec), was then added to cells. Cells were maintained at $37^{\circ} \mathrm{C}$ in a $5 \% \mathrm{CO}_{2}$ incubator for further experiments.

Current-clamp recording in transfected DRG neurons. Whole-cell configuration was obtained in voltage-clamp mode before starting current-clamp recording. The pipette solution contained (in mM): $140 \mathrm{KCl}$, 0.5 EGTA, $3 \mathrm{Mg}$-ATP, 5 HEPES, 30 dextrose, $\mathrm{pH}=7.3$ with $\mathrm{KOH}\left(310 \mathrm{mOsmoll}^{-1}\right)$. The extracellular bath solution contained (in $\mathrm{mM}$ ): $140 \mathrm{NaCl}, 3 \mathrm{KCl}, 2 \mathrm{MgCl}_{2}, 2 \mathrm{CaCl}_{2}, 15$ dextrose, 10 HEPES, $\mathrm{pH}=7.3$ with $\mathrm{NaOH}\left(315 \mathrm{mOsmol}^{-1}\right)$. Recording was performed on transfected nociceptive neurons, based on morphology with criteria of small diameter $(20-28 \mu \mathrm{m})$ and round cell bodies, which also exhibited GFP fluorescence. All recordings were performed 2 days after transfection. For CBZ experiments, neurons were treated with either CBZ $(30 \mu \mathrm{M})$ or DMSO as described above. RMP and seal stability for each neuron were evaluated during a 30-s-long period. Current threshold for action potential generation was determined by a series of $200 \mathrm{~ms}$ depolarizing currents in 5-pA increments. Repetitive firing frequency was examined using a series of 1 -s current steps from 25 to $500 \mathrm{pA}$ in 25 -pA increments. The interval between stimuli was $10 \mathrm{~s}$. For firing frequency measurement, spikes with overshoot beyond $0 \mathrm{mV}$ were counted as action potentials.

Data analysis. Data were analysed with Clampfit 9.2 (Molecular Devices) and OriginPro 8.5 (Microcal Software). For statistical analysis, if samples obeyed a normal distribution, Student's $t$-test was used. Non-parametric Mann-Whitney test was used when samples failed the normality test. Data was presented as means \pm s.e.m. Statistical significance was accepted when $P<0.05$.

\section{References}

1. Relieving Pain in America: A Blueprint for Transforming Prevention, Care, Education, and Research (Washington, DC, 2011).

2. Waxman, S. G. Neurobiology: a channel sets the gain on pain. Nature $\mathbf{4 4 4}$, 831-832 (2006).

3. Dib-Hajj, S. D., Cummins, T. R., Black, J. A. \& Waxman, S. G. From genes to pain: Na v 1.7 and human pain disorders. Trends Neurosci. 30, 555-563 (2007).

4. Dib-Hajj, S. D., Cummins, T. R., Black, J. A. \& Waxman, S. G. Sodium channels in normal and pathological pain. Annu. Rev. Neurosci. 33, 325-347 (2010).

5. Minett, M. S. et al. Distinct Nav1.7-dependent pain sensations require different sets of sensory and sympathetic neurons. Nat. Commun. 3, 791 (2012).

6. Cox, J. J. et al. An SCN9A channelopathy causes congenital inability to experience pain. Nature 444, 894-898 (2006).
7. Drenth, J. P. \& Waxman, S. G. Mutations in sodium-channel gene SCN9A cause a spectrum of human genetic pain disorders. J. Clin. Invest. 117, 3603-3609 (2007).

8. Han, C. et al. Sporadic onset of erythermalgia: a gain-of-function mutation in Nav1.7. Ann. Neurol. 59, 553-558 (2006).

9. Fischer, T. Z. et al. A novel Nav1.7 mutation producing carbamazepineresponsive erythromelalgia. Ann. Neurol. 65, 733-741 (2009).

10. Mantegazza, M., Curia, G., Biagini, G., Ragsdale, D. S. \& Avoli, M. Voltagegated sodium channels as therapeutic targets in epilepsy and other neurological disorders. Lancet Neurol. 9, 413-424 (2010).

11. Sheets, P. L., Jackson, 2nd J. O., Waxman, S. G., Dib-Haj, S. D. \& Cummins, T. R. A Nav1.7 channel mutation associated with hereditary erythromelalgia contributes to neuronal hyperexcitability and displays reduced lidocaine sensitivity. J. Physiol. 581, 1019-1031 (2007).

12. Doyle, D. A. et al. The structure of the potassium channel: molecular basis of $\mathrm{K}+$ conduction and selectivity. Science 280, 69-77 (1998).

13. Payandeh, J., Scheuer, T., Zheng, N. \& Catterall, W. A. The crystal structure of a voltage-gated sodium channel. Nature 475, 353-358 (2011).

14. Wu, S. \& Zhang, Y. LOMETS: a local meta-threading-server for protein structure prediction. Nucleic Acids Res. 35, 3375-3382 (2007).

15. Roy, A., Kucukural, A. \& Zhang, Y. I-TASSER: a unified platform for automated protein structure and function prediction. Nat. Protoc. 5, 725-738 (2010).

16. Zhang, J. \& Zhang, Y. GPCRRD: G protein-coupled receptor spatial restraint database for 3D structure modeling and function annotation. Bioinformatics 26, 3004-3005 (2010).

17. Zhang, Y. I-TASSER server for protein 3D structure prediction. BMC Bioinfo 9, 40 (2008).

18. Xu, J. \& Zhang, Y. How significant is a protein structure similarity with TMscore $=0.5$ ? Bioinformatics 26, 889-895 (2010).

19. Murzin, A. G., Brenner, S. E., Hubbard, T. \& Chothia, C. SCOP: a structural classification of proteins database for the investigation of sequences and structures. J. Mol. Biol. 247, 536-540 (1995).

20. Lampert, A., Dib-Hajj, S. D., Tyrrell, L. \& Waxman, S. G. Size matters: Erythromelalgia mutation S241T in Nav1.7 alters channel gating. J. Biol. Chem. 281, 36029-36035 (2006)

21. Dib-Hajj, S. D. et al. Gain-of-function mutation in Nav1.7 in familial erythromelalgia induces bursting of sensory neurons. Brain 128, 1847-1854 (2005).

22. Lampert, A. et al. A pore-blocking hydrophobic motif at the cytoplasmic aperture of the closed-state Nav1.7 channel is disrupted by the erythromelalgiaassociated F1449V mutation. J. Biol. Chem. 283, 24118-24127 (2008).

23. Yifrach, O. \& MacKinnon, R. Energetics of pore opening in a voltage-gated $\mathrm{K}(+)$ channel. Cell 111, 231-239 (2002).

24. Ranganathan, R., Lewis, J. H. \& MacKinnon, R. Spatial localization of the K+ channel selectivity filter by mutant cycle-based structure analysis. Neuron 16, 131-139 (1996).

25. DeCaen, P. G., Yarov-Yarovoy, V., Zhao, Y., Scheuer, T. \& Catterall, W. A. Disulfide locking a sodium channel voltage sensor reveals ion pair formation during activation. Proc. Natl Acad. Sci. USA 105, 15142-15147 (2008).

26. DeCaen, P. G., Yarov-Yarovoy, V., Sharp, E. M., Scheuer, T. \& Catterall, W. A. Sequential formation of ion pairs during activation of a sodium channel voltage sensor. Proc. Natl Acad. Sci. USA 106, 22498-22503 (2009).

27. Yarov-Yarovoy, V. et al. Structural basis for gating charge movement in the voltage sensor of a sodium channel. Proc. Natl Acad. Sci. USA 109, E93-102 (2012).

28. Rauer, H. et al. Structure-guided transformation of charybdotoxin yields an analog that selectively targets $\mathrm{Ca}(2+)$-activated over voltage-gated $\mathrm{K}(+)$ channels. J. Biol. Chem. 275, 1201-1208 (2000).

29. Lipkind, G. M. \& Fozzard, H. A. Molecular model of anticonvulsant drug binding to the voltage-gated sodium channel inner pore. Mol. Pharmacol. 78, 631-638 (2010).

30. Harty, T. P. et al. $\mathrm{Na}(\mathrm{V}) 1.7$ mutant A863P in erythromelalgia: effects of altered activation and steady-state inactivation on excitability of nociceptive dorsal root ganglion neurons. J. Neurosci. 26, 12566-12575 (2006).

31. Cestele, S. et al. Structure and function of the voltage sensor of sodium channels probed by a beta -scorpion toxin. J. Biol. Chem. 281, 21332-21344 (2006).

32. Yang, Y. et al. Molecular basis and structural insight of vascular K(ATP) channel gating by S-glutathionylation. J. Biol. Chem. 286, 9298-9307 (2011).

33. Yang, Y., Shi, W., Cui, N., Wu, Z. \& Jiang, C. Oxidative stress inhibits vascular K(ATP) channels by S-glutathionylation. J. Biol. Chem. 285, 38641-38648 (2010).

34. Roy, A., Xu, D., Poisson, J. \& Zhang, Y. A protocol for computer-based protein structure and function prediction. J. Vis. Exp. 3, e3259 (2011).

35. Zhang, Y. Template-based modeling and free modeling by I-TASSER in CASP7. Proteins 69(Suppl 8): 108-117 (2007).

36. Labro, A. J. et al. The S4-S5 linker of KCNQ1 channels forms a structural scaffold with the S6 segment controlling gate closure. J. Biol. Chem. 286, 717-725 (2011). 
37. Labro, A. J. et al. Kv channel gating requires a compatible S4-S5 linker and bottom part of S6, constrained by non-interacting residues. J. Gen. Physiol. 132, 667-680 (2008).

38. Choveau, F. S. et al. KCNQ1 channels voltage dependence through a voltagedependent binding of the S4-S5 linker to the pore domain. J. Biol. Chem. 286, 707-716 (2011).

39. Jensen, M. O. et al. Mechanism of voltage gating in potassium channels. Science 336, 229-233 (2012).

40. Sheets, M. F., Chen, T. \& Hanck, D. A. Lidocaine partially depolarizes the S4 segment in domain IV of the sodium channel. Pflugers Arch. 461, 91-97 (2011).

41. Capes, D. L., Arcisio-Miranda, M., Jarecki, B. W., French, R. J. \& Chanda, B. Gating transitions in the selectivity filter region of a sodium channel are coupled to the domain IV voltage sensor. Proc. Natl Acad. Sci. USA 109, 2648-2653 (2012).

42. Muroi, Y., Arcisio-Miranda, M., Chowdhury, S. \& Chanda, B. Molecular determinants of coupling between the domain III voltage sensor and pore of a sodium channel. Nat. Struct. Mol. Biol. 17, 230-237 (2010).

43. Catterall, W. A. From ionic currents to molecular mechanisms: the structure and function of voltage-gated sodium channels. Neuron 26, 13-25 (2000).

44. Nau, C. \& Wang, G. K. Interactions of local anesthetics with voltage-gated $\mathrm{Na}+$ channels. J. Membr. Biol. 201, 1-8 (2004).

45. Fozzard, H. A., Sheets, M. F. \& Hanck, D. A. The sodium channel as a target for local anesthetic drugs. Front. Pharmacol. 2, 68 (2011).

46. Lipkind, G. M. \& Fozzard, H. A. Molecular modeling of local anesthetic drug binding by voltage-gated sodium channels. Mol. Pharmacol. 68, 1611-1622 (2005).

47. Nau, C., Wang, S. Y., Strichartz, G. R. \& Wang, G. K. Point mutations at $\mathrm{N} 434$ in D1-S6 of mul $\mathrm{Na}(+)$ channels modulate binding affinity and stereoselectivity of local anesthetic enantiomers. Mol. Pharmacol. 56, 404-413 (1999).

48. Sheets, M. F. \& Hanck, D. A. Molecular action of lidocaine on the voltage sensors of sodium channels. J. Gen. Physiol. 121, 163-175 (2003).

49. Arcisio-Miranda, M., Muroi, Y., Chowdhury, S. \& Chanda, B. Molecular mechanism of allosteric modification of voltage-dependent sodium channels by local anesthetics. J. Gen. Physiol. 136, 541-554 (2010).

50. Zhang, J., Liang, Y. \& Zhang, Y. Atomic-level protein structure refinement using fragment-guided molecular dynamics conformation sampling. Structure 19, 1784-1795 (2011).

51. Zhang, J. \& Zhang, Y. A novel side-chain orientation dependent potential derived from random-walk reference state for protein fold selection and structure prediction. PLoS One 5, e15386 (2010).

52. Zhang, Y. \& Skolnick, J. SPICKER: a clustering approach to identify near-native protein folds. J. Comput. Chem. 25, 865-871 (2004).

53. Li, R. A. et al. Clockwise domain arrangement of the sodium channel revealed by (mu)-conotoxin (GIIIA) docking orientation. J. Biol. Chem. 276, 11072-11077 (2001)
54. Dudley, Jr. S. C. et al. mu-conotoxin GIIIA interactions with the voltage-gated $\mathrm{Na}(+)$ channel predict a clockwise arrangement of the domains. J. Gen. Physiol. 116, 679-690 (2000)

55. Zhang, Y. \& Skolnick, J. TM-align: a protein structure alignment algorithm based on the TM-score. Nucleic Acids Res. 33, 2302-2309 (2005).

56. Lossin, C., Wang, D. W., Rhodes, T. H., Vanoye, C. G. \& George, Jr. A. L. Molecular basis of an inherited epilepsy. Neuron 34, 877-884 (2002).

57. Cummins, T. R., Dib-Hajj, S. D. \& Waxman, S. G. Electrophysiological properties of mutant Nav1.7 sodium channels in a painful inherited neuropathy. J. Neurosci. 24, 8232-8236 (2004).

58. Estacion, M. et al. NaV1.7 gain-of-function mutations as a continuum: A1632E displays physiological changes associated with erythromelalgia and paroxysmal extreme pain disorder mutations and produces symptoms of both disorders. $J$. Neurosci. 28, 11079-11088 (2008).

59. Han, C. et al. Early- and late-onset inherited erythromelalgia: genotypephenotype correlation. Brain 132, 1711-1722 (2009).

\section{Acknowledgements}

We thank Dr Andrew Tan for comments on the manuscript, and Palak Shah and Bart Toftness for excellent technical assistance. This work was supported by the Medical Research Service and Rehabilitation Research Service, Department of Veterans Affairs and the Erythromelalgia Association (S.D.D-H. and S.G.W.). The Center for Neuroscience and Regeneration Research is a Collaboration of the Paralyzed Veterans of America with Yale University. J.Z. and Y.Z. are grateful for support from NSF Career Award (1027394) and NIGMS (GM083107 and GM084222).

\section{Author contributions}

Y.Y., S.D.D-H., M.E. and S.G.W. designed the study. Y.Y. performed the experiments. Y.Y., S.D.D-H., and M.E. analysed the data. J.Z. and Y.Z. provided new tools. L.T. provided new reagents. S.D.D-H. and S.G.W. supervised the research. Y.Y., S.D.D-H., M.E. and S.G.W wrote the paper with inputs from all authors.

\section{Additional information}

Supplementary Information accompanies this paper at http://www.nature.com/ naturecommunications

Competing financial interests: The authors declare no competing financial interests.

Reprints and permission information is available online at http://npg.nature.com/ reprintsandpermissions/

How to cite this article: Yang, Y. et al. Structural modeling and mutant cycle analysis predict pharmacoresponsiveness of a $\mathrm{Na}_{\mathrm{v}} 1.7$ mutant channel. Nat. Commun. 3:1186 doi: 10.1038/ncomms2184 (2012). 